\title{
CONVEXITY AND THE EXTERIOR INVERSE PROBLEM OF POTENTIAL THEORY
}

\author{
STEPHEN J. GARDINER AND TOMAS SJÖDIN
}

(Communicated by Juha M. Heinonen)

\begin{abstract}
Let $\Omega_{1}$ and $\Omega_{2}$ be bounded solid domains such that their associated volume potentials agree outside $\Omega_{1} \cup \Omega_{2}$. Under the assumption that one of the domains is convex, it is deduced that $\Omega_{1}=\Omega_{2}$.
\end{abstract}

\section{INTRODUCTION}

For any (positive Radon) measure $\mu$ with compact support in Euclidean space $\mathbb{R}^{N}(N \geq 2)$, we define the usual potential

$$
U^{\mu}(x)=\int U_{y}(x) d \mu(y) \quad\left(x \in \mathbb{R}^{N}\right),
$$

where $U_{y}(x)=|x-y|^{2-N}$ if $N \geq 3$, and $U_{y}(x)=\log (1 /|x-y|)$ if $N=2$. In the case where $\mu$ is the restriction of volume measure $\lambda$ to a bounded Borel set $A$, we will write $U^{A}$ in place of $U^{\left.\lambda\right|_{A}}$. A domain $\Omega$ in Euclidean space $\mathbb{R}^{N}$ is called solid if it is bounded, $(\bar{\Omega})^{\circ}=\Omega$ and the complement, $\bar{\Omega}^{c}$, of $\bar{\Omega}$ is connected.

A long-standing open question, known as the exterior inverse problem of potential theory, asks: if $U^{\Omega_{1}}=U^{\Omega_{2}}$ on $\left(\Omega_{1} \cup \Omega_{2}\right)^{c}$, where $\Omega_{1}$ and $\Omega_{2}$ are solid domains, does it follow that $\Omega_{1}=\Omega_{2}$ ? (The answer is "no" if we omit the word "solid", as is obvious from the example of a ball and a suitably chosen concentric annular domain of equal measure.) An early result on this problem, due to Novikov [6], says that the answer is "yes" if we require both $\Omega_{1}$ and $\Omega_{2}$ to be convex (or, more generally, starlike with respect to a common point). More recently, Shahgholian [7] proved that it is enough here for $\Omega_{1} \cap \Omega_{2}$ to be convex. Kondraškov [5] has shown that the answer to the question is also "yes" if one of the domains is a ball or an ellipsoid (cf. [1]; an elegant elementary proof for the case of a ball may be found in [9]). In this paper we show that convexity of one of the domains is sufficient to arrive at a positive answer.

Theorem 1. Let $\Omega_{1}$ be a solid domain and $\Omega_{2}$ be a convex domain, and let $\nu$ be a measure such that $\nu \geq\left.\lambda\right|_{\Omega_{2}}$ and $\nu\left(\Omega_{2}^{c}\right)=0$. If $U^{\Omega_{1}}=U^{\nu}$ on $\left(\Omega_{1} \cup \Omega_{2}\right)^{c}$, then $\Omega_{2} \subseteq \Omega_{1}$.

Corollary 2. Let $\Omega_{1}$ be a solid domain and $\Omega_{2}$ be a convex domain. If $U^{\Omega_{1}}=U^{\Omega_{2}}$ on $\left(\Omega_{1} \cup \Omega_{2}\right)^{c}$, then $\Omega_{1}=\Omega_{2}$.

Received by the editors February 19, 2007.

2000 Mathematics Subject Classification. Primary 31B20.

This research was supported by Science Foundation Ireland under Grant 06/RFP/MAT057. 
There is no implication in either direction between the corollary and the result of Shahgholian mentioned above. However, it is worth noting that Theorem 1 only imposes an additional hypothesis on one of the domains in question. Zalcman [9] has conjectured a stronger version of Corollary 2 in which $U^{\Omega_{1}}$ and $U^{\Omega_{2}}$ are only assumed to agree near infinity. The proof of Theorem 1 will be given in Section 3, following some preliminary material in Section 2 concerning the notion of partial balayage, on which it is based.

\section{Partial Balayage}

Let $a_{N}=\sigma_{N} \max \{1, N-2\}$, where $\sigma_{N}$ denotes the surface area of the unit sphere in $\mathbb{R}^{N}$, and let $q(x)=a_{N}|x|^{2} /(2 N)$. Thus $U^{\Omega}+q$ is harmonic on $\Omega$, for any bounded open set $\Omega$. If $\mu$ is a measure with compact support, it is easy to see that there is a greatest subharmonic minorant $s_{\mu}$, say, of $U^{\mu}+q$ on $\mathbb{R}^{N}$ (using Theorem 3.7 .5 of [2], for example). We need the following facts (see [3], [8]).

Theorem A. Let $\mu$ and $s_{\mu}$ be as above. Then:

(i) the function $s_{\mu}-q$ can be expressed as $U^{f \lambda}$, where $f: \mathbb{R}^{N} \rightarrow[0,1]$ is a Borel function with compact support;

(ii) the open set $\omega(\mu)=\left\{U^{\mu}>U^{f \lambda}\right\}$ is bounded, and $f \lambda=\left.\lambda\right|_{\omega(\mu)}+\left.\mu\right|_{\omega(\mu)^{c}}$.

The measure $f \lambda$ arising in Theorem $\mathrm{A}$ is called the partial balayage of $\mu$ onto $\lambda$, and will be denoted by $\widetilde{\mu}$. Obviously, $U^{\widetilde{\mu}} \leq U^{\mu}$. The decomposition formula in (ii) arises from the fact that $s_{\mu}$ must be harmonic on $\omega(\mu)$, by standard balayage arguments. It is clear from the lemma that if we define $\Omega(\mu)$ to be the largest open set $\Omega$ for which $(\lambda-\widetilde{\mu})(\Omega)=0$, then $\Omega(\mu)$ is bounded and contains $\omega(\mu)$, and

$$
\widetilde{\mu}=\left.\lambda\right|_{\Omega(\mu)}+\left.\mu\right|_{\Omega(\mu)^{c}} .
$$

The next result is a generalization of a fact due to Gustafsson (see pp. 205-206 of $[3])$.

Lemma 3. Let $\Omega_{1}$ and $\Omega_{2}$ be bounded open sets, where $\lambda\left(\partial \Omega_{2}\right)=0$, and let $\nu$ be a measure such that $\nu \geq\left.\lambda\right|_{\Omega_{2}}, \nu\left(\Omega_{2}^{c}\right)=0$, and $U^{\nu}$ is continuous on $\Omega_{2}$. If $U^{\nu}=U^{\Omega_{1}}$ on $\left(\Omega_{1} \cup \Omega_{2}\right)^{c}$, and we denote by $\eta$ the measure satisfying $U^{\eta}=\min \left\{U^{\Omega_{1}}, U^{\nu}\right\}$, then $\widetilde{\eta}=\left.\lambda\right|_{\Omega(\eta)}$.

Proof. Let $\Omega=\Omega(\eta)$ and

$$
\begin{aligned}
D_{1} & =\left\{U^{\Omega_{1}}<U^{\nu}\right\}, \quad D_{2}=\left\{U^{\Omega_{1}}>U^{\nu}\right\}, \quad S=\left\{U^{\Omega_{1}}=U^{\nu}\right\}, \\
A & =\left(\Omega_{1} \cap D_{1}\right) \cup\left(\Omega_{2} \cap D_{2}\right) \cup\left(\Omega_{1} \cap \Omega_{2}\right) .
\end{aligned}
$$

Then $A$ is an open set. Since $U^{\eta}=U^{\Omega_{1}}$ on $D_{1}, U^{\eta}=U^{\nu}$ on $D_{2}$, and

$$
U^{\eta}=U^{\Omega_{1} \cap \Omega_{2}}+\min \left\{U^{\Omega_{1} \backslash \Omega_{2}},\left.U^{\nu-\lambda}\right|_{\Omega_{1} \cap \Omega_{2}}\right\}
$$

we see that $\left.\eta\right|_{A} \geq\left.\lambda\right|_{A}$ and so $\left.\widetilde{\eta}\right|_{A}=\left.\lambda\right|_{A}$. It follows that $A \subseteq \Omega$. Since $\left(\Omega_{1} \cup \Omega_{2}\right)^{c} \subseteq$ $S$, we see that

$$
\Omega^{c} \subseteq A^{c} \subseteq A_{1} \cup A_{2} \cup \partial \Omega_{2},
$$

where

$$
A_{1}=\Omega_{1}^{c} \cap\left(D_{1} \cup S\right) \text { and } A_{2}=\bar{\Omega}_{2}^{c} \cap\left(D_{2} \cup S\right) .
$$

On $A_{1} \cap \Omega^{c}$ we have $U^{\widetilde{\eta}}=U^{\eta}=U^{\Omega_{1}}$, and since $U^{\widetilde{\eta}}$ and $U^{\Omega_{1}}$ both belong to the Sobolev space $W_{\text {loc }}^{2,2}\left(\mathbb{R}^{N}\right)$, we have $\widetilde{\eta}\left(A_{1} \cap \Omega^{c}\right)=\left.\lambda\right|_{\Omega_{1}}\left(A_{1} \cap \Omega^{c}\right)=0$. Since 
$U^{\widetilde{\eta}}=U^{\eta}=U^{\nu}$ on $A_{2} \cap \Omega^{c}$ and $U^{\nu}$ is harmonic on $\bar{\Omega}_{2}^{c}$, we similarly have $\widetilde{\eta}\left(A_{2} \cap \Omega^{c}\right)=$ 0 . Hence $\widetilde{\eta}\left(\Omega^{c}\right)=0$, in view of $(3)$ and the fact that $\lambda\left(\partial \Omega_{2}\right)=0$. The result now follows by applying (1) to the measure $\eta$.

We denote a typical point $x$ of $\mathbb{R}^{N}$ by $\left(x^{\prime}, x_{N}\right)$, where $x^{\prime} \in \mathbb{R}^{N-1}$ and $x_{N} \in \mathbb{R}$, and define

$$
W_{+}=\left\{x: x_{N}>0\right\}, \quad W_{-}=\left\{x: x_{N}<0\right\} \text { and } H=\left\{x: x_{N}=0\right\} .
$$

The following result is due to Gustafsson and Sakai [4]. We give a short proof here for the sake of completeness.

Lemma 4. Let $\mu$ be a measure with compact support contained in $W_{-} \cup H$ and let $A=\left\{x^{\prime}:\left(x^{\prime}, 0\right) \in \Omega(\mu) \cap H\right\}$. Then there is a continuous function $g: A \rightarrow(0, \infty)$, continuously vanishing at $\partial A$, such that

$$
\Omega(\mu) \cap W_{+}=\left\{\left(x^{\prime}, x_{N}\right): x^{\prime} \in A \text { and } 0<x_{N}<g\left(x^{\prime}\right)\right\} .
$$

Proof. Let $u=U^{\mu}-U^{\widetilde{\mu}}$. Thus $u \geq 0$. We may assume, by means of a limiting argument, that $\operatorname{supp} \mu \subset W_{-}$, and so $u$ is continuously differentiable on an open set containing $W_{+} \cup H$. Let $\bar{u}(x)=u\left(x^{\prime},-x_{N}\right)$. We note that $U^{\mu}-\bar{u}+q$ is subharmonic on $W_{+}$, and $U^{\widetilde{\mu}}+q$ is subharmonic on all of $\mathbb{R}^{N}$. Since

$$
U^{\mu}-\bar{u}+q=U^{\mu}-u+q=U^{\widetilde{\mu}}+q \text { on } H,
$$

the function

$$
v= \begin{cases}\max \left\{U^{\mu}-\bar{u}+q, U^{\widetilde{\mu}}+q\right\} & \text { on } W_{+}, \\ U^{\widetilde{\mu}}+q & \text { on } W_{-} \cup H\end{cases}
$$

is a subharmonic minorant of $U^{\mu}+q$. Thus $v \leq U^{\widetilde{\mu}}+q$ by the definition of $U^{\tilde{\mu}}$, whence $U^{\mu}-\bar{u} \leq U^{\widetilde{\mu}}$ on $W_{+}$and so $u \leq \bar{u}$ there. It follows that $\partial u / \partial x_{N} \leq 0$ on $H$.

Let $\Omega_{+}=\Omega(\mu) \cap W_{+}$. Since $u=0$ on $\omega(\mu)^{c}$, and so on $\Omega(\mu)^{c}$, and since every point of $\partial \Omega(\mu)$ is the limit of some sequence of points of Lebesgue density of $\Omega(\mu)^{c}$, we see that $|\nabla u|=0$ on $\partial \Omega_{+} \cap W_{+}$. We note from (1) that $\Delta u$ is constant in $\Omega_{+}$, so the function $\partial u / \partial x_{N}$ is harmonic there, and hence $\partial u / \partial x_{N} \leq 0$ on $\Omega_{+}$, by the maximum principle. Further, since $u$ is nonconstant in each component of $\Omega_{+}$, and $u=0$ on $W_{+} \backslash \Omega_{+}$, we actually have $\partial u / \partial x_{N}<0$ on $\Omega_{+}$. We now define

$$
g\left(x^{\prime}\right)=\sup \left\{t>0:\left(x^{\prime}, t\right) \in \Omega_{+}\right\} \quad\left(x^{\prime} \in A\right) .
$$

Clearly $\Omega(\mu) \cap W_{+}$lies under the graph of $g$. Conversely, if $\left(x^{\prime}, x_{N}\right)$ lies under the graph of $g$ and $x_{N}>0$, then $u\left(x^{\prime}, x_{N}\right)>0$ and so $\left(x^{\prime}, x_{N}\right) \in \omega(\mu) \subseteq \Omega(\mu)$. Thus (4) holds.

It remains to check that $g$ is continuous and vanishes at $\partial A$. In fact, since $\Omega(\mu)$ is open and

$$
\left\{x^{\prime}: g\left(x^{\prime}\right)>c\right\}=\left\{x^{\prime}:\left(x^{\prime}, c\right) \in \Omega(\mu)\right\} \quad(c>0),
$$

it is clear that $g$ is lower semicontinuous. On the other hand, if we apply the result of the previous paragraph with hyperplanes of varying orientation, we see that each point of $\partial \Omega_{+} \cap W_{+}$is the vertex of a vertical cone lying in $\Omega(\mu)^{c}$, and so $g$ is also upper semicontinuous. In fact, $g$ continuously vanishes at $\partial A$, since we can apply the preceding reasoning with $H$ replaced by a slightly lower hyperplane. 


\section{Proof of Theorem 1}

Let $\Omega_{1}, \Omega_{2}$ and $\nu$ be as in the statement of Theorem 1 . We begin by observing that we may assume $U^{\nu}$ to be continuous on $\Omega_{2}$. To see this, let $\left(\omega_{n}\right)$ be an increasing sequence of regular open sets with union $\Omega_{2}$ such that $\bar{\omega}_{n} \subset \omega_{n+1}$ for each $n$, and let $\nu_{n}=\left.(\nu-\lambda)\right|_{\omega_{n} \backslash \omega_{n-1}}$, where $\omega_{0}=\emptyset$. If we define $u_{n}=U^{\nu_{n}}$ on $\omega_{n+1}^{c}$, and extend $u_{n}$ to $\mathbb{R}^{N}$ by solving the Dirichlet problem on $\omega_{n+1}$, then the function $U^{\Omega_{2}}+\sum u_{n}$ is continuous on $\Omega_{2}$, equals $U^{\nu}$ on $\Omega_{2}^{c}$ and can be expressed as $U^{\nu^{\prime}}$ with $\nu^{\prime} \geq\left.\lambda\right|_{\Omega_{2}}$ and $\nu^{\prime}\left(\Omega_{2}^{c}\right)=0$.

Now let $\eta$ be as in Lemma 3, and let $\Omega=\Omega(\eta)$. As we noted earlier, it follows from (2) that $\Omega_{1} \cap \Omega_{2} \subseteq \Omega$. We will suppose that

$$
\lambda\left(\Omega_{1} \backslash \Omega\right)>0
$$

with a view to reaching a contradiction.

Let $D=\Omega \cup \Omega_{2}$. Our first step is to show that

$$
U^{\widetilde{\eta}}=U^{\Omega}=U^{\nu} \quad \text { on } \quad D^{c} .
$$

To see this, we note that $U^{\widetilde{\eta}}=U^{\eta}$ on $\Omega^{c}$, since $\omega(\eta) \subseteq \Omega$, and $U^{\widetilde{\eta}}=U^{\Omega}$, by Lemma 3. On $D^{c} \backslash \Omega_{1}$, which coincides with $\left(\Omega_{1} \cup \Omega_{2}\right)^{c} \cap \Omega^{c}$, we thus have $U^{\nu}=$ $U^{\Omega_{1}}=U^{\eta}=U^{\widetilde{\eta}}$. The nonnegative function $U^{\Omega_{1}}-U^{\Omega}$ is superharmonic on $\Omega_{1}$. It cannot be constant on $\Omega_{1}$, in view of (5), so it is strictly positive there. Hence $U^{\Omega_{1}}>U^{\Omega}=U^{\widetilde{\eta}}=U^{\eta}=U^{\nu}$ on $D^{c} \cap \Omega_{1}$. We have now proved (6).

Let

$$
E=\Omega_{2} \backslash \Omega \text { and } \mu=\nu+\left.\lambda\right|_{E},
$$

whence $D=\Omega \cup E$. Clearly $U^{\Omega}=U^{\widetilde{\eta}} \leq U^{\eta} \leq U^{\nu}$, so

$$
U^{D}=U^{\Omega}+U^{E} \leq U^{\nu}+U^{E}=U^{\mu},
$$

and from (6) we see that

$$
U^{D}=U^{\Omega}+U^{E}=U^{\nu}+U^{E}=U^{\mu} \text { on } D^{c} .
$$

We note from (7) that $U^{D}+q$ is a subharmonic minorant of $U^{\mu}+q$, so $U^{D} \leq U^{\widetilde{\mu}} \leq$ $U^{\mu}$. The nonnegative function $U^{\widetilde{\mu}}-U^{D}$ vanishes on $D^{c}$, by (8), and hence on $\mathbb{R}^{N}$, since it is subharmonic on $D$. Thus

$$
\widetilde{\mu}=\left.\lambda\right|_{D} \text { and } D \subseteq \Omega(\mu) .
$$

Further,

$$
0=(\lambda-\widetilde{\mu})(\Omega(\mu))=\lambda(\Omega(\mu) \backslash D) \geq \lambda\left(\left(\Omega(\mu) \backslash \bar{\Omega}_{2}\right) \backslash \Omega\right)=(\lambda-\widetilde{\eta})\left(\Omega(\mu) \backslash \bar{\Omega}_{2}\right),
$$

so $\Omega(\mu) \backslash \bar{\Omega}_{2} \subseteq \Omega \subseteq D$. In view of (9) we thus see that

$$
D \backslash \bar{\Omega}_{2}=\Omega(\mu) \backslash \bar{\Omega}_{2} \text {. }
$$

We now claim that $\partial \Omega_{1} \subset \bar{D}$. For, if this were not the case, we could choose an open ball $B \subset \bar{D}^{c}$ that intersects $\partial \Omega_{1}$. Since $U^{\Omega_{1}} \geq U^{\eta} \geq U^{\widetilde{\eta}}=U^{\nu}$ on $D^{c}$, by (6), the function $U^{\Omega_{1}}-U^{\nu}$, which is nonnegative and superharmonic on $B$ and attains the value 0 on $B \backslash \Omega_{1}$, must vanish identically on $B$. This leads to a contradiction, as $B \cap \Omega_{1} \neq \emptyset$.

Next we claim that $\Omega_{1} \backslash \bar{\Omega}_{2} \subset D$. For, if there were a point $y \in \Omega_{1} \backslash\left(D \cup \bar{\Omega}_{2}\right)$, then we could assume (by choosing a suitable coordinate system) that the closest point of $\bar{\Omega}_{2}$ to $y$ is 0 , and $y=\left(0^{\prime},|y|\right)$. Let $y_{0}=\left(0^{\prime}, t_{0}\right)$, where $t_{0}=\sup \left\{t:\left(0^{\prime}, t\right) \in \Omega_{1}\right\}$. Then $t_{0}>|y|$ and $y_{0} \in \partial \Omega_{1} \subset \bar{D} \subseteq \overline{\Omega(\mu)}$, by the preceding paragraph and (9). 
Also, $y \in \Omega(\mu)^{c}$, by (10). Since $\operatorname{supp} \mu=\bar{\Omega}_{2} \subset W_{-} \cup H$, Lemma 4 now yields the desired contradiction.

In view of the previous paragraph we see that

$$
\lambda\left(\Omega_{1} \backslash \Omega\right)=\lambda\left(\left(\Omega_{1} \cup \Omega_{2}\right) \backslash D\right) \leq \lambda\left(\partial \Omega_{2}\right)=0,
$$

which contradicts (5). Thus $\lambda\left(\Omega_{1} \backslash \Omega\right)=0$ and so $\Omega_{1} \subseteq \Omega$, by the definition of $\Omega$. Since $\lambda\left(\Omega_{1}\right)=\lambda(\Omega)$, and $\Omega_{1}$ is solid, it follows that $\Omega=\Omega_{1}$. Hence $U^{\nu}-U^{\Omega}$ is a nonnegative superharmonic function on $\bar{\Omega}^{c}$ which attains the value 0 , so $U^{\nu}=$ $U^{\Omega}=U^{\Omega_{1}}$ there, and therefore $\lambda\left(\Omega_{2} \backslash \bar{\Omega}_{1}\right)=0$. It follows that $\Omega_{2} \subset \bar{\Omega}_{1}$, and so $\Omega_{2} \subseteq\left(\bar{\Omega}_{1}\right)^{\circ}=\Omega_{1}$, as required.

The corollary is immediate, since $\lambda\left(\Omega_{1}\right)=\lambda\left(\Omega_{2}\right)$ in this case.

\section{REFERENCES}

[1] D. Aharonov, M. M. Schiffer and L. Zalcman, Potato kugel, Israel J. Math. 40 (1981), 331-339. MR654588 (83d:31002)

[2] D. H. Armitage and S. J. Gardiner, Classical potential theory. Springer Monographs in Mathematics. Springer, London, 2001. MR1801253 (2001m:31001)

[3] B. Gustafsson, On quadrature domains and an inverse problem in potential theory, J. Analyse Math. 55 (1990), 172-216. MR1094715 (92c:31013)

[4] B. Gustafsson and M. Sakai, Properties of some balayage operators, with applications to quadrature domains and moving boundary problems, Nonlinear Anal. 22 (1994), 1221-1245. MR1279981 (95h:31007)

[5] A. V. Kondraškov, On the uniqueness of the reconstruction of certain regions from their exterior gravitational potentials (Russian) Ill-posed Mathematical Problems and Problems of Mathematical Geophysics, Novosibirsk (1976), pp. 122-129.

[6] P. S. Novikoff, Sur le problème inverse du potentiel, C. R. (Dokl.) Acad. Sci. URSS (N.S.) 18 (1938), 165-168.

[7] H. Shahgholian, Convexity and uniqueness in an inverse problem of potential theory, Proc. Amer. Math. Soc. 116 (1992), 1097-1100. MR1137234 (93b:31008)

[8] T. Sjödin, On the structure of partial balayage, Nonlinear Anal. 67 (2007), 94-102. MR2313881

[9] L. Zalcman, Some inverse problems of potential theory, Integral geometry (Brunswick, Maine, 1984), pp. 337-350, Contemp. Math., 63, Amer. Math. Soc., Providence, RI, 1987. MR876329 (88e:31012)

School of Mathematical Sciences, University College Dublin, Belfield, Dublin 4, IRELAND

E-mail address: stephen.gardiner@ucd.ie

School of Mathematical Sciences, University College Dublin, Belfield, Dublin 4, IRELAND

E-mail address: tomas.sjodin@ucd.ie 